\title{
Series of Cases with Iatrogenic Nerve Injury
}

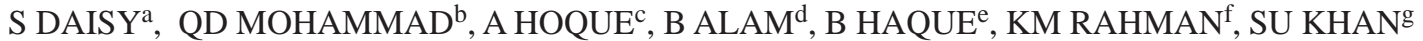

\section{Summary: \\ After introduction of EMG at Dhaka Medical Collage on January 01, 2006, a total of 415 cases referred to EMG laboratory for electrophysiological evaluation over a period of two years}

(January 01, 2006 to December 31, 2007). Among these, 7 cases diagnosed as iatrogenic nerve injuries. The subtypes of iatrogenic nerve injuries were:

1. Accessory nerve injury: three, 2. Femoral nerve injury: one, 3. Sciatic nerve injury: one,

\section{Introducton:}

Nerve injury produces considerable disability which is often irreversible. Several patients with peripheral nerve injuries were evaluated in our EMG laboratory at Neurology Dept, Dhaka Medical College, Dhaka for localization of their lesion and for assessment of their disabilities. EMG plays very crucial role to localize the lesion, to determine the severity of lesion, duration of lesion, and to observe the sign of reinnevation that is important for prognosis.

Materials and Methods:

This retrospective study was conducted at EMG laboratory under Neurology Department of Dhaka Medical Collage. Study period was two years (January 01, 2006 to December 31, 2007). Both inpatient and

\footnotetext{
a. Dr. Selina Daisy, MD. (USA)

b. Professor Dr. Quazi Deen Mohammad, FCPS (Medicine), MD (Neuro), Fellow Neurology (America)

c. Dr. Azharul Hoque, FCPS (Medicine), MD (Neurology)

d. Dr. Badrul Alam, MD (Neurology)

e. Dr. Badrul Haque, M.B.B.S, Dip in Neuro.

f. Dr. Kazi Mohibur Rahman, MD (Neurology)

g. Dr. Sharif Uddin Khan, MD (Neurology)

Address of Correspondence: Dr. Selina Daisy, MD. (USA), Associate Professor of Neurology, Department of Neurology, Dhaka Medical College, Dhaka, Bangladesh. Mailing address: daisyselina@yahoo.com.
}

Received: 23 September, 2008

Accepted: 5 April, 2010
4. Lumbar sacral plexus injury: one, 5. Combined sciatic and femoral nerve injury: one

In order to investigate the causes, diagnosis \& prevention of iatrogenic nerve injuries; we have reviewed 7 cases of iatrogenic nerve injuries. The peripheral nerve injuries occurred due to lack of proper awareness of medical personals. These injuries are iatrogenic injuries so it is useful to review the mode of injuries and means of prevention.

(J Bangladesh Coll Phys Surg 2010; 28: 121-124)

outdoor patient were referred to EMG laboratory with various diagnoses.

All patients' clinical information along with some laboratory data collected and stored in Excel program and the data was reviewed. The inclusion criteria were nerve injury due to physician or medical assistant handling. Nerve injury by other means like road traffic accident, accidental injuries etc were all excluded.

After history was taken and a directed physical examination performed, a routine nerve conduction study (NCS) followed by needle electromyogram (EMG) were done on all patients.

Needle EMG cannot be planned or properly interpreted without knowledge of underlying nerve. So nerve conduction study (NCS) followed by needle electromyogram (EMG) are done together.

Nerve selected for study depends on the distribution of the patient's symptoms and sign and the differential diagnosis.

Case \#1.

History \& physical examination:

A 16 year old girl was referred for progressive dropping of right shoulder and also pain \& weakness of right shoulder joint. There was history of cervical node tuberculoses and which was diagnosed by lymph node biopsy an age of 8 years \& was treated appropriately.

Examination showed right shoulder drop with mild scapular winging when arm was abducted. 
Rest of the shoulder muscles, sternocleidomastoid muscle, reflexes \& sensation were intact.

NCS \& EMG diagnosis: Distal spinal accessory neuropathy (axonal)

Discussion: The most common site of injury to the spinal accessory nerve occurs in the region of the posterior cervical triangle, where the nerve runs superficially ${ }^{1}$. A lesion at this site results in shoulder drop and atrophy of the trapezium muscle, with sparing of the sternocleidomastoid muscle ${ }^{1,2,3}$, and 4 .

Anatomy ${ }^{1,2,3,4}$ : The spinal accessory nerve originates from the C1-C4 roots, ascending through the foramen magnum then returning via the jugular foramen. The nerve first innervates the sternocleidomastoid muscle before running over the posterior cervical triangle to innervate the trapezius muscle. There are no cutaneous sensory innervations. Accessory nerve injury produces considerable disability ${ }^{6}$. The nerve is most frequently damaged as a complication of radical neck dissection, cervical lymph node biopsy and other surgical procedures around the neck $6,7,8$,

We had 3 patients, all of them had cervical lymph node biopsy so surgeons should be very careful during the operation to prevent distal portion of spinal accessory nerve injury. Even if the injury had happened, it should be recognized as early possible to prevent disability. Early surgical repair has the greatest chance of recovery and success ${ }^{6}$.

\section{Case \# 2:}

History \& physical examination:

A 50 year old obese female presented with right foot drop for 6 month. And was unable to stand without support on her right leg or get up from sitting posture. Her problem started 6 months later after she had hysterectomy, which was done for menorrhagia. After this operation she developed progressive weakness of her right leg and while standing her knee would buckle up. And since then she could not stand on her right foot and gradually symptom progressed to present condition. When seen 6 month later, her neurology examination, showed a complete foot drop or no movement of ankle or great toe, hip abduction, hip flexion, hip extension, hip lateral rotation, and knee flexion, knee extension $(0-1 / 5)$. Sensory exam showed reduced sensation throughout her right lower extremity. Ankle \& knee reflexes were absent.

NCS\& EMG diagnosis: Chronic lesion (axonal) of the right lumbosacral plexus.

\section{Discussion:}

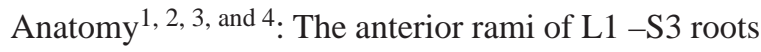
come together to from the lumbosacral plexus, from which all major lower extremity nerves are derived.

Lumbosacral plexopathy can he caused by structural and nonstructural lesion like surgical procedure especially when retractors are used. Most likely this had happened to this patient, who had undergone pelvic surgery for hysterectomy.

\section{Case \# 3:}

History \& physical examination:

A 20 year old boy presents with slight dragging of right leg. He had a history of high fever when he was 8 years old and was given 10 days IM shot at buttock, which was given by village doctor.

Local examination of his buttock region showed mild atrophy of his gluteus maximums muscle.

On examination all were normal except muscle bulk below knee was mildly reduced on right side with some weakness of right ankle and toe dorsiflexion (3/5) as foot inversion. Ankle and toe planer flexion, knee extension, and all movements around the hip were symmetric. Sensory examination showed reduced sensation of right leg below knee except medial side of leg which was normal.

NCS \& EMG Diagnosis: Chronic sciatic (axonal) neuropathy.

Discussion: Sciatic nerve injury is not so common in western countries. But in our country it is relatively common. It is due to failure to give IM shot at proper location i.e. upper outer quadrant. So the nerve can be injured by misplaced shot. Intramuscular injection to gluteal muscle should be strictly localized to upper outer quadrant.

\section{Case \# 4:}

History \& physical examination:

A 58 year old nondiabetic, non hypertensive obese man presented with progressive difficulty in standing or keep weight on right leg for 6 weeks after undergoing elective cardiac catheterization for his chest pain. The patient 
also noted that his leg would occasionally buckle, and he had nearly fallen several times. He also experienced a pins and needles sensation in the front of the thigh, radiating to the inner calf. There was no significant pain in the leg. There were no symptoms in his left leg

On examination muscle bulk and tone were normal. Strength test showed normal strength in all muscles including hip flexion, ankle dorsiflexion, thigh adduction \& knee extension were normal but when the patient was subsequently asked to arise from the kneeling position, he was unable to do so leading with the right leg, but could easily do so on the left side. (To demonstrate subtle weakness this test is done.) Sensory examination showed mildly reduced sensation over the anterior thigh and medial calf and sole of the foot were normal.

NCS \& EMG Diagnosis: Sabacute femoral neuropathy (both demyelination \& axonal lesion).

\section{Discussion:}

Isolated lesions of the femoral nerve are not often seen in the electromyography (EMG) laboratory. More common are lesions of the lumber plexus or L2-4 nerve roots, which may present with similar symptoms and signs of femoral neuropathy. Especially in milder cases, differentiating between these three types of lesions may be difficult. The EMG serves two major roles in suspected lesions of the femoral nerve: first to localize the lesion, which often suggests the correct diagnosis, and second, to assess the severity and degree of axonal loss, which has direct implications for the prognosis and duration of disability ${ }^{1}$.

Anatomy 1, 2, 3 and 4: The femoral nerve is derived from lumber plexus and receives innervations from L 2, L 3 and $\mathrm{L} 4$ nerve roots. Muscular branches are given off to the psoas and then to the iliacus muscle, before the nerve runs beneath the inguinal ligament. It then divides into motor \& sensory branches. Motor branches innervate the sartorius and pectineus muscles and the four heads of quadriceps. Sensory branches of the femoral nerve supply medial and anterior thigh and medial calf (Saphenous). Most cases of femoral neuropathy result from positioning or compression during abdominal or pelvic surgery ${ }^{10}$. Retractions used during surgery may result in compression of the femoral nerve against the pelvis. Compression can also occur at the inguinal ligament if patient is placed in lithotomy position for prolong period during surgery. Otherwise isolated femoral neuropathies are uncommon. Iatrogenic femoral neuropathy can occur in the inguinal region from misguided femoral catheterization as a consequence of hematoma formation, which happened to our patient. Femoral neuropathy may also occur in patients with diabetes mellitus, presumably from nerve infarction, this usually occurs in the setting of a more widespread polyradiculoplexopathy (I.e. diabetic amyotrophy).

The nerve conduction studies and EMG demonstrate a postganglionic lesion of the femoral nerve, most likely at the inguinal region. The preserved hip flexion strength correlates with the normal EMG examination of the Iliopsoas. This finding is important in excluding a lesion proximal to the inguinal ligament. By suggesting that the lesion is at the inguinal region ligament, the EMG is helpful in determining that the most likely etiology of the neuropathy is at the inguinal region from misguided femoral catheterizations as a consequence of hematoma formation. So physician doing catheterization should know this iatrogenic injury of femoral nerve, so that they can avoid the injury.

\section{Case \# 5:}

History and physical examination:

A five year old boy presents with unable to stand on his left leg. He had a history of left leg fracture at mid thigh after a fall from a height. It was treated with cast for several weeks. After the cast was removed he could walk with slight dragging of his left leg. His grandma felt a slight elevation of the bone at the fracture site so she took her grandson to a village doctor to fix the slight elevation of the fracture site.

The village doctor tied a rope tightly around the thigh at the elevation site and told her to keep the tie for 12 hours.

After few hours the boy started complaining pain at left leg \& wanted to take off the rope. He was given some sweets to cool him down. And then the tie was removed after 12 hours. After removing the tie the boy could not bear weight on that leg and kept his knee semi flexed.

On examination there was mild atrophy of left leg with mild flexion of left knee with complete foot drop. Toe, ankle dorsiflexion as well as knee flexion were absent. Hip flexion, extension, abduction \& adduction were normal. Deep tendon reflex was absent. Sensory was absent in whole leg. 
NCS \& EMG diagnosis: Severe injury (axonal) of left femoral, obturator \& sciatic nerve with its branches i.e. tibial \& peroneal nerve

\section{Discussion:}

Anatomy 1, 2, 3, and 4: - The sciatic nerve is derived from the L4 - S3 roots, carrying fibres that will eventually become the tibial and common personal nerves. It leaves the pelvis through the greater sciatic foramen under the piriformis muscle accompanied by the other branches of the lumbosacral plexus (inferior and superior gluteal nerves and posterior cutaneous nerve of the thigh. The sciatic nerve next runs between the ischial tuberosity and the greater trochanter of the femur covered by the gluteus maximus. The knee flexors including the semimembranosus, semitendinosus and long and short head of biceps femoris and the lateral division of adductor magnus, are all supplied by the sciatic nerve. The sciatic nerve terminates in the popliteal fossa where it divides into the common peroneal and tibial nerves, supplying all motor and sensory innervations below the knee, with the exception of sensation over the medial calf and foot (saphenous sensory territory)

Sciatic neuropathy is distinctly uncommon and usually suggests a structural etiology. One of the most common causes of sciatic neuropathy is tumor (neurofibroma, schwannoma, neurofibrosarcoma, lipoma, lymphoma), which is usually imaged quite well as a mass lesion on computed tomography (CT) or magnetic imaging (MRI) scanning. Damage of the sciatic nerve can also occur from trauma or as a result of a penetrating injury, such as gunshot and knife wounds. Damage to the sciatic nerve may also occur iatrogenically from misplaced intramuscular buttock injections, as in our case no. 3 . Here in our patient damage happened due to pressure from the tie.

Cases reported where peripheral nerve injury occurred due to the pressure of a restraint buckle causing a postoperative motor and sensory deficit. But our case was due to ignorance, that a healed fracture site was thicker from surroundings.

\section{Conclusion:}

Iatrogenic injury is preventable. So knowing proper anatomy and also disease process, it can be easily avoided. Even if the injury had happened, it should be recognized as early as possible to prevent disability. Early surgical repair has the greatest chance of recovery and success. It is the responsibility of physician, attending doctors, medical assistance and especially surgeons. And their careful manipulation during operation and knowledge of regional anatomy were the key to prevent nerve injuries. To recognize the injury and to localize the lesion, nerve conduction study (NCS) along with electromyogram (EMG) should be done. Awareness of iatrogenic injury and its consequences should be emphasized for prevention. And early diagnosis and management improves the outcome.

\section{Acknowledgement:}

We are thankful to the principal of Dhaka Medical Collage \& the Director of Dhaka Medical Collage Hospital for allowing us to publish this study. We are also thankful to the concerned family patient for their cooperation.

\section{References:}

1. Preston DC, Shapiro BE, Electromyography and Neuromuscular disorders 2005 Elsevier ButterworthHeinemann.

2. Brazis PW, Masden JC, Biller J, Localization in clinical Neurology 1996 Little Brown \& Co, ISBN O-316-09992-9

3. Patten J Neurological Differential Diagnosis, $2^{\text {nd }}$ Edition, Springer - Verlay Berlin Heidelberg New York, ISBN 3-54019937-3

4. Kimura J , Electrodiagnosis in Diseases of Nerve and Muscles: Principles \& Practice, $3^{\text {rd }}$ Edition , Oxford University Press 2001, ISBN 0-19-512977-6

5. Song X, He C, Zhang G Iatrogenic nerve injuries and its repair, Medline. 1998 May; 12(3):133-4.

6. London J, London NJ, Kay SP. Iatrogenic accessory nerve injury. Ann R Coll Surg Engl. 1996 Mar; 78( 2):146-50.

7. Yavuzer G, Tuncer S: Accessory nerve injury as a complication of cervical lymph biopsy. Am J Phys Med Rehabil 2001; 80:622-623

8. Haiukewych GJ, Scaduto J, Herscovici D Jr, Sanders RW, DiPasquale T: Iatrogenic nerve injury in acetabular fracture surgery: a comparison of monitored and unmonitored procedure. J Orthop Trauma. 2002May;16(5):297-301.

9. Hudson AR. Hunter GA, Waddell JP: Iatrogenic femoral nerve injuries. Can J Surg. 1972 Jan; 22(1):62-6. 